\title{
Investigation of in situ gelling alginate formulations as a sustained release vehicle for co-precipitates of dextromethrophan and Eudragit S $\mathbf{1 0 0}$
}

\author{
GAMAL MOHAMED EL MAGHRABY ${ }^{1,2}$ \\ EHAB MOSTAFA ELZAYAT ${ }^{1, *}$ \\ FARS KAED ALANAZI ${ }^{1,3}$ \\ ${ }^{1}$ Department of Pharmaceutics \\ College of Pharmacy, King Saud University \\ Riyadh 11451, P.O. Box 2457 \\ Saudi Arabia
}

${ }^{2}$ Department of Pharmaceutical Technology College of Pharmacy, University of Tanta Tanta, Egypt

\section{${ }^{3}$ Alkayyali Research Chair for Pharmaceutical Industries \\ College of Pharmacy, King Saud University}

Accepted September 16, 2013

\begin{abstract}
Alginate vehicles are capable of forming a gel matrix in situ when they come into contact with gastric medium in the presence of calcium ions. However, the gel structure is $\mathrm{pH}$ dependent and can break after gastric emptying, leading to dose dumping. The aim of this work was to develop modified in situ gelling alginate formulations capable of sustaining dextromethorphan release throughout the gastrointestinal tract. Alginate solution $(2 \%, \mathrm{~m} / \mathrm{m})$ was used as a vehicle for the tested formulations. Solid matrix of the drug and Eudragit S 100 was prepared by dissolving the drug and polymer in acetone. The organic solvent was then evaporated and the deposited solid matrix was micronized, sieved and dispersed in alginate solution to obtain candidate formulations. The release behavior of dextromethorphan was monitored and evaluated in a medium simulating the gastric and intestinal pH. Drug-polymer compatibility and possible solid-state interactions suggested physical interaction through hydrogen bonding between the drug and the polymer. A significant decrease in the rate and extent of dextromethorphan release was observed with increasing Eudragit $S$ 100 concentration in the prepared particles. Most formulations showed sustained release profiles similar to that of a commercial sustained-release liquid based on ion exchange resin. The release pattern indicated strict control of drug release both under gastric and intestinal conditions, suggesting the potential advantage of using a solid dispersion of drug-Eudragit S 100 to overcome the problem of dose dumping after the rupture of the $\mathrm{pH}$ dependent alginate gels.
\end{abstract}

Keywords: dextromethorphan, solid dispersion, in situ gelling, alginate gels

\footnotetext{
* Correspondence; e-mail: ezayat@ksu.edu.sa
} 
The development of liquid oral sustained-release formulations using the in situ gelling technique has gained much interest recently. These formulations maintain the convenience of sustained release drug delivery and help eliminate the problems associated with the administration of oral solid dosage forms especially for pediatrics, geriatrics and patients who may suffer from swallowing difficulties (1). Different polymers have been employed to develop such systems utilizing several drugs (2-5). In these systems, an aqueous solution of the polymer containing the drug in dispersed or dissolved form forms a gel when it comes in contact with a physiological trigger like temperature change, $\mathrm{pH}$ change or ionic interaction (6). Alginate based in situ gelling systems, which induce in situ gelation in the stomach, are most widely used in this field. Their polymeric solutions contain divalent ions, complexed with sodium citrate and are expected to break upon exposure to the acidic environment of the stomach to release free divalent ions (mostly $\mathrm{Ca}^{2+}$ ), which cause cross-linking and gelation of the orally administered solutions (7). Optimal quantities of calcium chloride and sodium citrate that maintain fluidity of the formulation before administration and result in gelation when the formulation is added to simulated gastric fluid (SGF) ( $\mathrm{pH}$ 1.2) were determined previously (8). However, these systems undergo immediate phase transition from gel to sol on increasing the $\mathrm{pH}$ as a result of gastric emptying. Accordingly, if premature gastric emptying takes place, dose dumping will take place. Consequently, sodium alginate formulations may need further modification aimed at retarding drug release for a sufficient period of time especially in the small intestine. It is therefore useful to develop modified in situ gelling formulations to sustain drug release from alginate gels.

Methacrylic resins (Eudragits ${ }^{\circledR}$ ) appear particularly attractive due to their high chemical stability, good compatibility with different drugs and a large variety of products with different physicochemical characteristics present at the market (9). Eudragits are widely used as tablet adjuvants and coating polymers (9). Sias da Fonseca (10) evaluated the potentiality to control drug release of Eudragit $S 100{ }^{\circledR}$ microparticles organized at the nanoscopic scale by assembling polymeric nanocapsules at the surface of drug-loaded xerogels. Their formulation showed satisfactory gastro-resistance drug release of less than $3 \%$ in gastric medium within about $60 \mathrm{~min}$. They concluded that the new system was able to control the release of the hydrophilic drug model, sodium diclofenac.

This study combined the alginate based in situ gelling system with a $\mathrm{pH}$-dependent polymer to control drug release. Eudragit ${ }^{\circledR}$ S 100 with pH-dependent solubility was used at different concentrations to prepare a solid matrix of the drug and the polymer by solid dispersion. The resulting matrix was micronized and sieved. The particles were then dispersed in $2 \%$ alginate solution to develop candidate formulations suitable for sustained delivery of dextromethorphan (DM).

Dextromethorphan is a methyl ether of the dextrorotatory (D) isomer of levorphanol, a codeine analog. Its chemical name is (+)-3-methoxy-17-methyl-9 $\alpha, 13 \alpha, 14 \alpha$-morphinan hydrobromide monohydrate (11). It is a highly effective and widely used antitussive drug (12). DM is used clinically in the form of salt, DM hydrobromide. At therapeutic doses, its onset of action is 15 to 30 minutes and its duration of action is 5 to 6 hours (13). Due to the short duration of DM action (i.e., short half-life), it has to be administrated at a frequency of 3 to 4 times a day. Therefore, preparation of DM in a sustained release formulation should improve patient compliance, reduce the number of doses and reduce the cost associated with treating cough symptoms. 


\section{EXPERIMENTAL}

\section{Materials}

Eudragit $^{\circledR}$ S 100 was a generous gift from Degussa, Kirschenalle, Germany. Sodium alginate, low molecular mass (Lot 1993F, $150 \mathrm{cP}$ for a $1 \%$ solution) was purchased from ICN Biomedicals Inc., Germany. Dextromethorphan hydrobromide (DM) was generously donated by the TABUK Pharmaceutical Company Tabuk, KSA. Calcium chloride anhydrous and trisodium citrate were obtained from WINLAB, GEMINI House, UK. Methanol HPLC grade and disodium hydrogen phosphate were obtained from BDH, Prolabo, Poole, England. Potassium dihydrogen phosphate was obtained from Merck, Darmstadt, Germany. All other reagents were of analytical grade.

\section{Preparation of solid dispersions}

Solid dispersions were prepared according to the composition presented in Table I. DM and Eudragit S100 were dissolved in acetone and organic solvent was removed at 50 ${ }^{\circ} \mathrm{C}$. The co-evaporates were left in a Petri dish for 24 hours at ambient temperature for complete dryness. The resulting matrix was powdered and sieved to obtain a particle size not exceeding $350 \mu \mathrm{m}$.

Table I. The composition of the prepared binary systems of DM and Eudragit S 100 in different ratios $(\mathrm{m} / \mathrm{m})$ and drug content values after recovery

\begin{tabular}{cccc}
\hline Binary systems & DM & Eudragit S 100 & Drug content $^{\mathrm{c}}$ \\
\hline $\mathrm{MC}^{\mathrm{a}} / \mathrm{M} 1^{\mathrm{b}}$ & 1 & 1 & $101.91 \%(2.181)$ \\
$\mathrm{MC}^{\mathrm{a}} / \mathrm{M}^{\mathrm{b}}$ & 1 & 2 & $99.98 \%(1.16)$ \\
$\mathrm{MC}^{\mathrm{a}} / \mathrm{M}^{\mathrm{b}}$ & 1 & 3 & $101.57 \%(2.067)$ \\
$\mathrm{MC}^{\mathrm{a}} / \mathrm{M}^{\mathrm{b}}$ & 1 & 4 & $103.40 \%(3.69)$ \\
\hline
\end{tabular}

a Solid microparticles (control formulations).

b Solid microparticles dispersed in alginate vehicle $(2 \%, m / V)$.

c Values between brackets are SD $(n=3)$.

\section{Differential scanning calorimetry}

Thermograms of the samples (DM, Eudragit S 100, physical mixture, prepared particles) were recorded using differential scanning calorimetry (DSC) (DSC-60, Shimadzu, Japan). Samples equivalent to approximately $2.4 \mathrm{mg}$ of the drug were loaded into aluminum pans and the lids were crimped using a Shimadzu crimper. The thermal behavior of each sample was monitored under nitrogen at a heating rate of $10^{\circ} \mathrm{C} \mathrm{min}-1$, covering temperature ranges of $25-200{ }^{\circ} \mathrm{C}$. The instrument was calibrated with an indium standard. Data analysis was conducted using the TA 60WS thermal analysis software. The 
G. M. El Maghraby et al.: Investigation of in situ gelling alginate formulations as a sustained release vehicle for co-precipitates of dextromethrophan and Eudragit S 100, Acta Pharm. 64 (2014) 29-44.

following parameters were calculated: melting point $\left(T_{\text {onset }}\right)$ and enthalpy $(\Delta H$, calculated as the area under the transition peak normalized to the sample mass).

\section{Fourier transform infrared spectroscopy}

The Fourier transform infrared (FTIR) spectra of DM, Eudragit S 100, the prepared particles $(1: 4, \mathrm{~m} / \mathrm{m})$ and their physical mixture were recorded using an FTIR spectrophotometer (FTIR Nicolet-380, Thermo Fisher Scientific, WI, USA). Samples were mixed with potassium bromide (spectroscopic grade) and compressed into disks using a hydraulic press before scanning from 4000 to $600 \mathrm{~cm}^{-1}$. The data were analyzed using IR Solution software (version 1.10) (14).

\section{Powder X-ray diffraction}

Powder X-ray diffraction patterns of the drug, Eudragit S 100, physical mixture and the prepared particles were obtained with the aid of a wide-angle $X$-ray diffractometer (Rigaku Ultima IV X-Ray Diffractometer, Japan). The instrument was operated so as to conduct a full scan $\left(2 \theta\right.$ ranging from 0 to $\left.150^{\circ}\right)$ with the counts being accumulated for 1 second after each step (15).

\section{Determination of drug content}

The drug content of the prepared particles was determined spectrophotometrically $(\lambda=278 \mathrm{~nm})$. A predetermined mass of the solid matrix of Eudragit S 100 and DM (equivalent to $120 \mathrm{mg}$ of $\mathrm{DM}$ ) was dissolved in $50 \mathrm{~mL}$ methanol with sonication. After complete dissolution, the solution was suitably diluted with methanol before spectroscopic analysis at $278 \mathrm{~nm}$. Preliminary studies showed that the investigated polymer dilution range used did not interfere with DM absorbance at $278 \mathrm{~nm}$. All the experiments were conducted in triplicate.

\section{Preparation of in situ gelling systems}

In a previous study (16), solutions containing different concentrations $(1-4 \%, m / V)$ of alginate were evaluated for their rheological behavior and release profile. The most suitable concentration was found to be $2 \%(\mathrm{~m} / \mathrm{V})$ (designated as A2), which was used as a standard vehicle for all formulations in this work. The selection of this alginate concentration was based on the fact that it was the highest concentration controlling drug release and retaining formulation fluidity.

Briefly, alginate solution was prepared by adding a specified amount of alginate to $1 \%$ $(m / V)$ potassium dihydrogen phosphate solution (to adjust $\mathrm{pH}$ to below 7 to avoid dissolution of Eudragit S 100) containing $0.45 \%(\mathrm{~m} / \mathrm{V})$ sodium citrate and $0.15 \%(\mathrm{~m} / \mathrm{V})$ calcium chloride and heating to $60{ }^{\circ} \mathrm{C}$ under stirring (3). After cooling to below $40{ }^{\circ} \mathrm{C}$, the prepared particles were dispersed into the resulting alginate dispersion to produce homogenously dispersed formulations M1, M2, M3 and M4. Control formulations MC1, MC2, MC3 and MC4 are the prepared particles used as such without being dispersed in alginate or any other vehicle, Table I. 
G. M. El Maghraby et al.: Investigation of in situ gelling alginate formulations as a sustained release vehicle for co-precipitates of dextromethrophan and Eudragit S 100, Acta Pharm. 64 (2014) 29-44.

\section{Rheology of in situ gelling systems}

Rheological properties of all formulations were determined using a Brookfield viscometer RVDV-II+ (Brookfield Engineering Laboratories Inc., USA) with a small sample adapter at ambient temperature. A typical run comprised changing of angular velocity from 0.5 to $100 \mathrm{rpm}$ at a controlled ramp rate with a wait of $6 \mathrm{~s}$ at each speed (17). An average of the three readings was used to calculate the viscosity.

\section{Determination of in vitro drug release}

The method of evaluation of drug release from enteric coated systems was adopted according to USP 24. Release experiments employed a USP dissolution apparatus (Model: UDT-804, LOGAN Inst. Corp., USA) with a paddle stirrer maintained at $50 \mathrm{rpm}$. The release medium was $500 \mathrm{~mL}$ of simulated gastric fluid without enzymes $\left(0.1 \mathrm{~mol} \mathrm{~L}^{-1} \mathrm{HCl}\right.$, $\mathrm{pH}$ 1.2) and the temperature was maintained at $37 \pm 0.2{ }^{\circ} \mathrm{C}$. Test formulations $(10 \mathrm{~mL})$ were loaded into a Petri dish $(2.7 \mathrm{~cm}$, internal diameter) before immersion into the dissolution vessel containing release medium without much disturbance. Samples $(5 \mathrm{~mL})$ were collected at predetermined time intervals (5, 15, 30, 60, 90 and 120 minutes). Fresh release medium was replenished for each sample. The samples were filtered through a $0.45 ? \mathrm{~m}$ filter immediately after collection and the filtrate was analyzed for drug content using the HPLC method (see below). After the last sample $(2 \mathrm{~h})$, the $\mathrm{pH}$ of the release medium was adjusted to 6.8 to simulate the intestinal $\mathrm{pH}$ (USP 24). This was achieved by addition of $200 \mathrm{~mL}$ of $0.3 \mathrm{~mol} \mathrm{~L}-1$ dibasic sodium phosphate and $25 \mathrm{~mL}$ of $1 \mathrm{~mol} \mathrm{~L}^{-1}$ sodium hydroxide. Sampling was then continued for another 4 hours.

Cumulative amounts of the drug released (expressed as percent of the total drug added) were plotted as a function of time to produce the drug release profiles. The release efficiency (RE) was calculated from the area under the release curve at time $t$ (determined using the non-linear trapezoidal rule) and expressed as a percentage of the area of the rectangle described by $100 \%$ release in the same time (18). The results were compared to the release data of the commercial formulation (Delsym ${ }^{\circledR}$ suspension). In addition, the release data for each phase was fitted to different kinetic models to determine the release kinetics. This included fitting the data to zero order, first order and the Higuchi diffusion system. Each study was conducted in triplicate.

\section{Dextromethorphan assay}

Drug concentration in each sample was determined using the previously developed HPLC method of analysis (19). The study employed a high pressure liquid chromato-

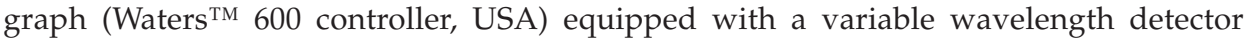
(SPD-10 AV, Shimadzu, Kyoto, Japan) and an automatic sampling system (Waters ${ }^{\mathrm{TM}} 717$ Plus Autosampler, USA). The whole system was under computer control. Separation was performed on a reversed phase column $15 \mathrm{~cm} \times 3.9 \mathrm{~mm}$ (i.d.) $\mathrm{C} 18, \mu$ Bondapak $^{\mathrm{TM}}$, Waters $^{\mathrm{TM}}$, with an average particle size of $10 \mu \mathrm{m}$. The mobile phase comprised methanol and $0.1 \mathrm{~mol} \mathrm{~L}^{-1}$ potassium dihydrogen phosphate buffer $(45: 55, V / V)$, flowing at $1.2 \mathrm{~mL}$ $\mathrm{min}^{-1}$ after being adjusted to $\mathrm{pH} 3$ with phosphoric acid. The column effluent was monitored at $220 \mathrm{~nm}$ and the chromatographic data analysis was performed with the 
G. M. El Maghraby et al.: Investigation of in situ gelling alginate formulations as a sustained release vehicle for co-precipitates of dextromethrophan and Eudragit S 100, Acta Pharm. 64 (2014) 29-44.

Millennium ${ }^{\mathrm{TM}}$ Program (Waters, USA). Filtered samples were loaded into the HPLC vials before injecting $30 \mu \mathrm{L}$ into HPLC.

\section{Statistical analysis}

One-way analysis of variance (ANOVA) was performed using the SPSS 18 software ${ }^{\circledR}$ to compare the mean values of the drug released from different formulations. Fisher's least significant difference procedure (LSD) was used to perform pairwise comparisons of means (20). Fisher's LSD is a method used for comparing treatment group means after the ANOVA null hypothesis of equal means has been rejected. The level of confidence was set at $95 \%$.

\section{RESULTS AND DISCUSSION}

\section{Solid state characterization of binary systems}

Differential scanning calorimetry. - Fig. 1 shows the DSC curves of DM, Eudragit S 100 , their physical mixture (1:4) and the co-evaporates (MC1, MC2, MC3 and MC4). Pure $\mathrm{DM}$ produced a single endothermic peak at $116^{\circ} \mathrm{C}$. This indicates the crystallinity of the drug. The recorded melting point value is in accord with literature values (21). Pure Eudragit $\mathrm{S} 100$ showed a broad endotherm between 32.2 and $94.6{ }^{\circ} \mathrm{C}$, which can be attributed to the moisture content of the sample (22). Appearance of such a peak in other formulae can be attributed to evaporation of water and/or solvent. The thermogram of Eudragit S 100 did not show any melting transition for the polymer in the tested tempe-

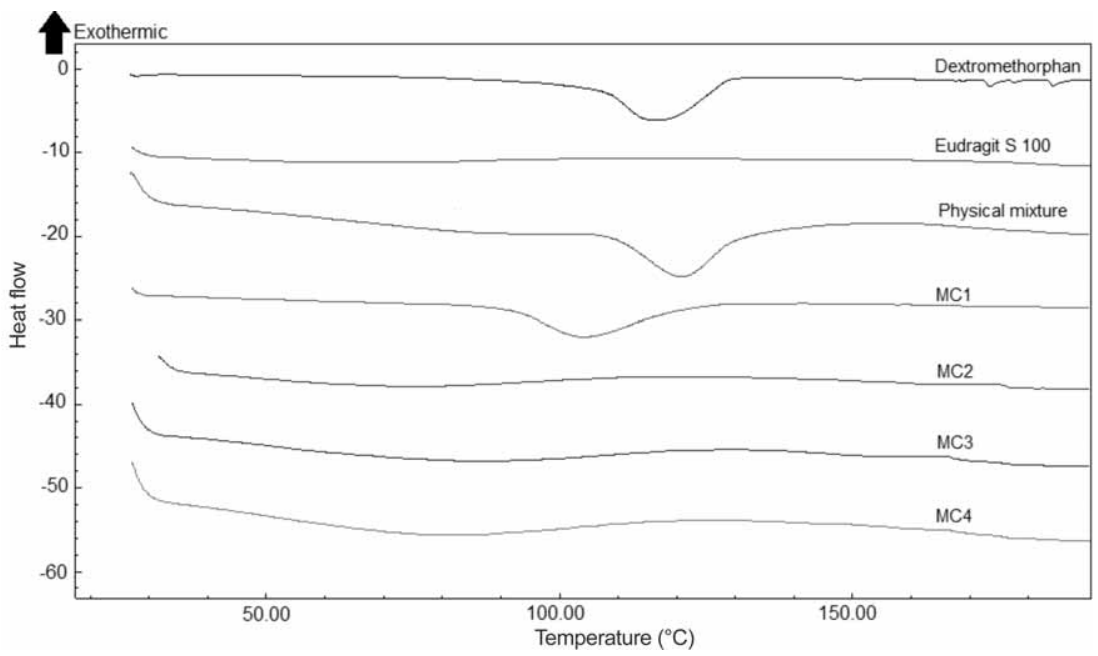

Fig. 1. The DSC curves of DM - Eudragit S 100 micropaticles. The curves from top to bottom are DM, Eudragit S 100, physical mixture, MC1, MC2, MC3 and MC4, respectively. 
rature range (20). The thermogram of the physical mixture showed the characteristic peak of the drug indicating no interaction with the polymer. For the prepared particles, the thermograms showed initial reduction in $T_{\mathrm{m}}$ of the endothermic peak of the drug in case of MC1, which contained the polymer at 1:1 mass ratio, compared to pure drug. Further increase in polymer concentration in the particles (MC2, MC3 and MC4) resulted in complete disappearance of the endothermic peak of the drug. This indicates loss of drug crystallinity or a possible interaction between DM and Eudragit S 100 (23). This will be clarified further in the following sections.

Fourier transform infrared spectroscopy. - The FTIR spectrum of pure DM (Fig. 2) showed characteristic absorption bands, which occurred at $3297 \mathrm{~cm}^{-1}$ corresponding to $\left(\mathrm{NH}^{+}\right.$ stretching vibration in the tertiary amine group of the drug), $2933 \mathrm{~cm}^{-1}$ corresponding to (C-H stretching), $1615 \mathrm{~cm}^{-1}$ corresponding to (C=C stretching), 1600-1450 $\mathrm{cm}^{-1}$ corresponding to (C-C aryl stretching) and $1300-900 \mathrm{~cm}^{-1}$ corresponding to (C-N and C-O stretching) (24). This is in agreement with the previously recorded spectra of the pure drug (21). The IR region from 1400 to $600 \mathrm{~cm}^{-1}$, termed the fingerprint region, usually contains a large number of unassigned vibrations. In the finger print region, correlation of an individual band with a specific functional group cannot usually be made with accuracy (24). The FTIR spectrum of Eudragit $S 100$ showed characteristic bands of $\mathrm{C}=\mathrm{O}$ vibrations of esterified carboxyl groups at $1735 \mathrm{~cm}^{-1}$. The spectrum also showed a very distinctive $\mathrm{O}-\mathrm{H}$ band, which begins at about $3330 \mathrm{~cm}^{-1}$ and slopes into the aliphatic $\mathrm{C}-\mathrm{H}$ absorption band at $2900-3000 \mathrm{~cm}^{-1}$. This spectrum correlates with the previously recorded one (25). The FTIR spectrum of the physical mixture showed the characteristic $\left(\mathrm{NH}^{+}\right.$ stretching band) of the drug at $3297 \mathrm{~cm}^{-1}$. It also showed the characteristic $\mathrm{C}=\mathrm{O}$ band of the polymer at $1735.64 \mathrm{~cm}^{-1}$. This indicates that no interaction occurred between the drug and the polymer in the physical mixture of ratio $(1: 4, \mathrm{~m} / \mathrm{m})$.

For MC4, some changes were recorded in the absorption bands around the wavelength range $4000-1500 \mathrm{~cm}^{-1}$. The prominent absorption band $\left(\mathrm{NH}^{+}\right.$stretching) of the drug disappeared in the solid dispersion. The binary solid dispersion MC4 showed a new broad band at $3422 \mathrm{~cm}^{-1}$. The characteristic $\mathrm{C}=\mathrm{O}$ absorption band of the polymer was significantly diminished. In addition, the fingerprint region was altered for both the drug and the polymer. It can be concluded that this is not the usual spectrum associated with either pure drug or polymer.

Formation of new chemical bonds would be expected to result in emergence of additional absorption bands or alterations in the wave number position (26). Hence, these findings can imply that dextromethorphan in the solid dispersion exists in the positively charged form $\left(\mathrm{NH}^{+}\right.$group of drug in solid dispersion), which interacts with the carbonyl group of the carboxylic group of Eudragit S 100 in the solid dispersion. This kind of ionic association is referred to as a salt bridge and it significantly reduces the $\mathrm{NH}^{+}$stretching (21). This emphasized the occurrence of interaction between the drug and the polymer.

X-ray diffraction study. - The powder X-ray diffraction (XRD) patterns of pure DM, Eudragit S 100, physical mixture and the prepared particles are shown in Fig. 3. The diffractogram of DM showed a number of sharp peaks of D-value 13.58, 5.604, 5.18, 3.914 and 3.8145 corresponding to DM crystals. The XRD pattern of Eudragit S 100 was typical of an amorphous substance, without any detectable intense peak in its diffractogram. 
G. M. El Maghraby et al.: Investigation of in situ gelling alginate formulations as a sustained release vehicle for co-precipitates of dextromethrophan and Eudragit S 100, Acta Pharm. 64 (2014) 29-44.

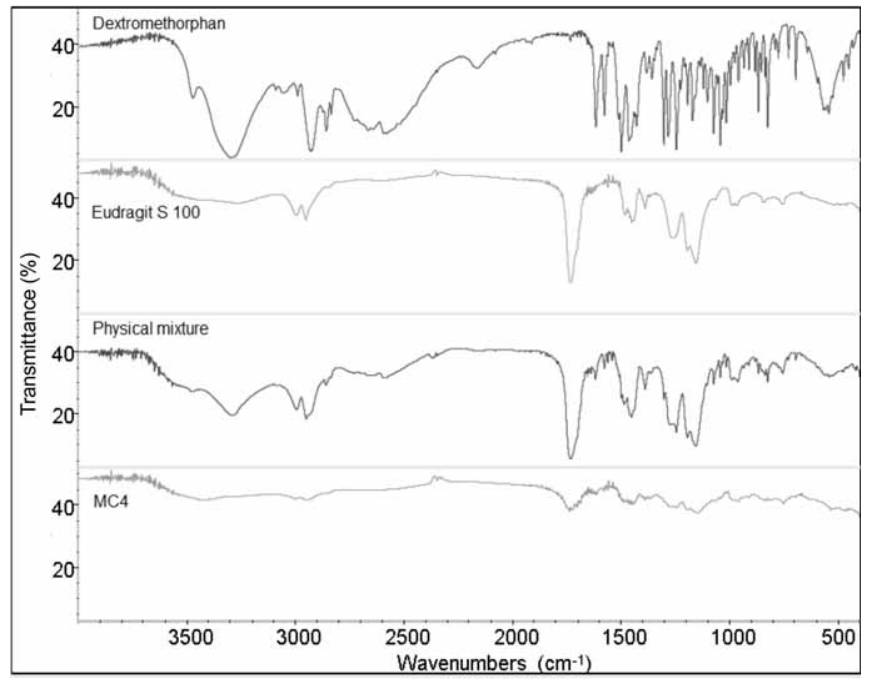

Fig. 2. Comparison of the FTIR spectra of DM, Eudragit S 100, physical mixture and MC4, from the top to the bottom.

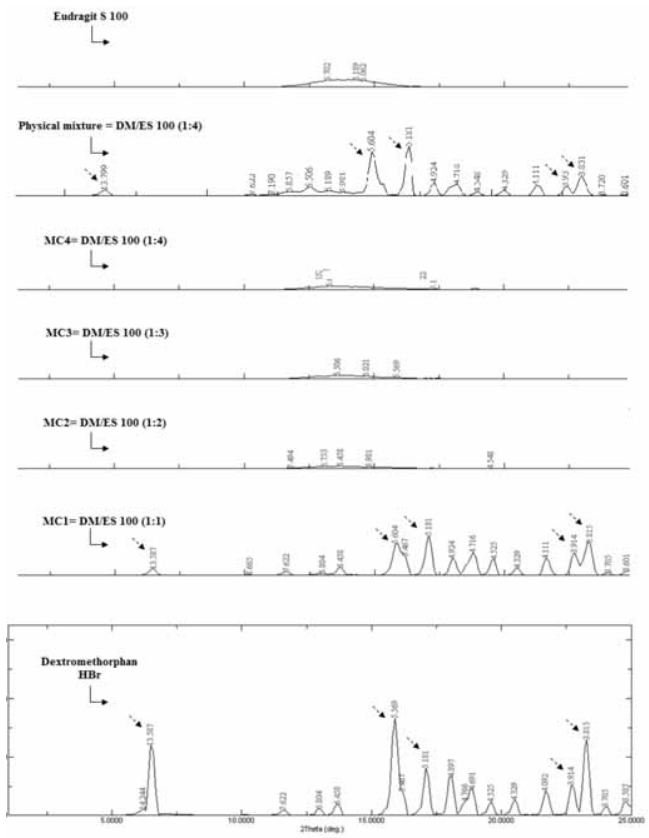

Fig. 3. Powder X-ray diffraction patterns of Eudragit S 100, Physical mixture, MC1, MC2, MC3, MC4 and DM from the top to the bottom. 
G. M. El Maghraby et al.: Investigation of in situ gelling alginate formulations as a sustained release vehicle for co-precipitates of dextromethrophan and Eudragit S 100, Acta Pharm. 64 (2014) 29-44.

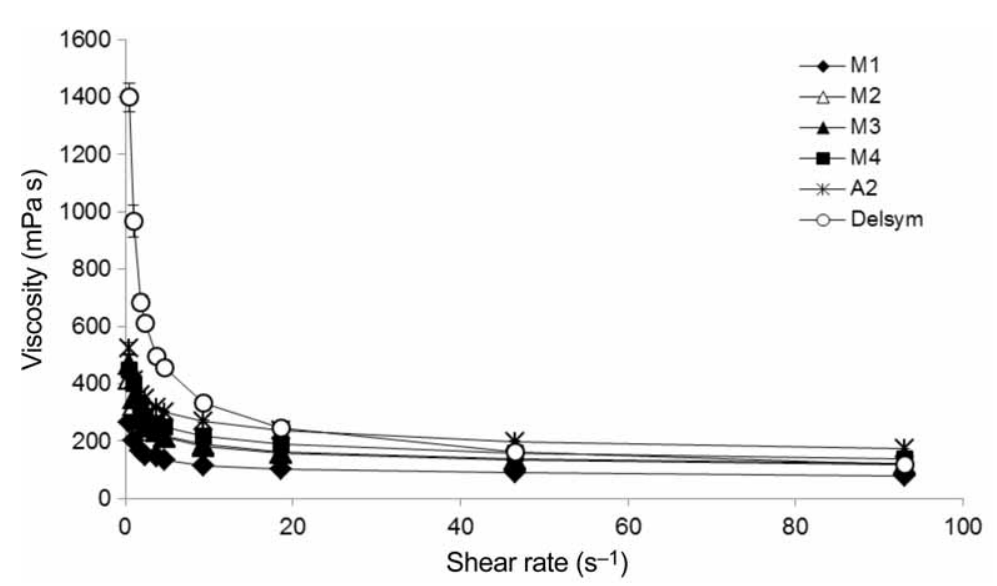

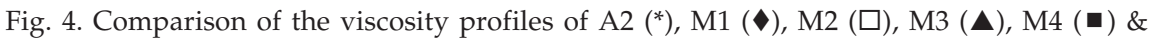
Delsym ${ }^{\circledR}$ suspension $(\bigcirc)$ as a function of shear rate. Each value is the mean $\pm \mathrm{SE}$ of three determinations.

The physical mixture X-ray diffraction pattern showed the characteristic peaks of the drug, indicating no interaction with the polymer.

Intensities of crystalline diffraction peaks of the drug in its solid dispersions were decreased noticeably with MC1. The peaks were diminished or completely absent with the other formulae, MC2, MC3 and MC4. Disappearance of diffraction peaks indicates the absence of crystalline structure.

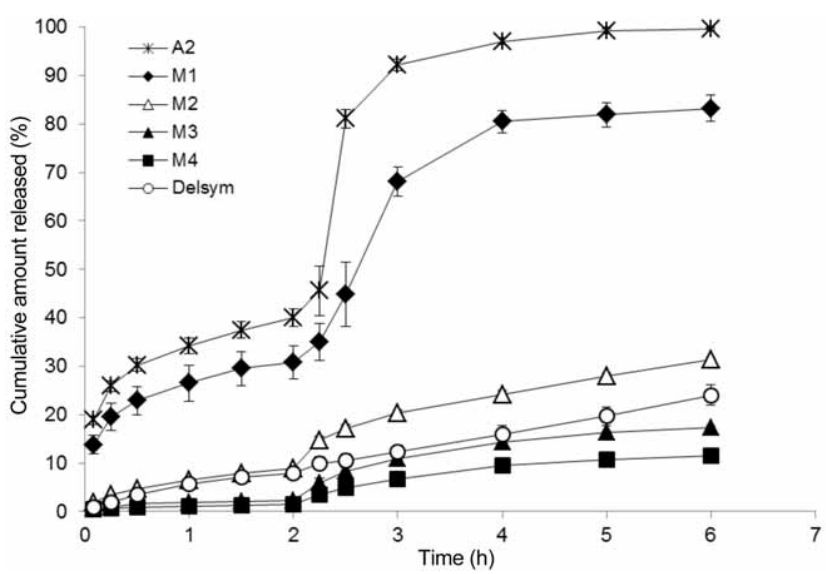

Fig. 5. In vitro release profiles of dextromethorphan hydrobromide from A2 (*), M1 (•), M2 ( $\square)$, M3 $(\boldsymbol{\Delta})$, M4 ( $)$ \& Delsym ${ }^{\circledR}$ suspension $(\bigcirc)$ plotted as cumulative \% released against time. Release was into simulated gastric fluid, $\mathrm{pH} 1.2$, for a period of $2 \mathrm{~h}$ and subsequently into simulated intestinal fluid, $\mathrm{pH} 6.8$, for a period of $4 \mathrm{~h}$. Each value is the mean $\pm \mathrm{SE}$ of three determinations. 
G. M. El Maghraby et al.: Investigation of in situ gelling alginate formulations as a sustained release vehicle for co-precipitates of dextromethrophan and Eudragit S 100, Acta Pharm. 64 (2014) 29-44.

Determination of drug content. - The drug content was determined to evaluate the homogeneity of distribution of the drug in the binary solid dispersion. The results revealed drug content values lying in the range of 95 to $105 \% \mathrm{~m} / \mathrm{m}$ as listed in Table I, indicating homogenous distribution of the drug in the prepared mixtures.

Rheological behavior of the in situ gelling systems. - The fluidity of liquid oral formulations should be maintained to assure the ease of administration and swallowing of the

Table II. The coefficient of determinations $\left(\mathrm{R}^{2}\right)$ for all the formulations after being fitted to Zero-order, First-order, Higuchi model and Korsmeyer-Peppas model

\begin{tabular}{|c|c|c|c|c|c|c|c|}
\hline \multicolumn{8}{|c|}{ Release analysis } \\
\hline \multirow{2}{*}{$\begin{array}{l}\text { Formulation } \\
\text { code }\end{array}$} & \multirow{2}{*}{ Phase } & \multicolumn{3}{|c|}{$R^{2}$} & \multirow{2}{*}{$n$} & \multirow{2}{*}{$\mathrm{RE}^{\mathrm{a}}$} & \multirow{2}{*}{$f_{2}{ }^{b}$} \\
\hline & & Zero-order & First-order & Higuchi & & & \\
\hline \multirow{2}{*}{ A2 } & SGF & 0.8831 & 0.8035 & 0.9679 & 0.23 & 33.1 & \multirow{2}{*}{$\sim$} \\
\hline & SIF & 0.785 & 0.7351 & $\underline{0.7998}$ & $\sim$ & 83.1 & \\
\hline \multirow{2}{*}{ M1 } & SGF & 0.8754 & 0.7917 & $\underline{0.9652}$ & 0.2504 & 25.56 & \multirow{2}{*}{20.14} \\
\hline & SIF & 0.904 & 0.8542 & $\underline{0.9245}$ & $\sim$ & 71.12 & \\
\hline \multirow{2}{*}{ M2 } & SGF & 0.9594 & 0.845 & $\underline{0.9991}$ & 0.4769 & 6.22 & \multirow{2}{*}{63.69} \\
\hline & SIF & 0.9821 & 0.9448 & $\underline{0.9934}$ & 0.4839 & 23.6 & \\
\hline \multirow{2}{*}{ M3 } & SGF & $\mathrm{NA}^{\mathrm{c}}$ & NA & NA & 0.443 & 1.74 & \multirow{2}{*}{71.31} \\
\hline & SIF & 0.917 & 0.8332 & $\underline{0.9476}$ & 0.5101 & 13.11 & \\
\hline \multirow{2}{*}{ M4 } & SGF & NA & NA & NA & 0.3564 & 1.07 & \multirow{2}{*}{59.51} \\
\hline & SIF & 0.9249 & 0.8404 & $\underline{0.9544}$ & 0.5964 & 8.48 & \\
\hline \multirow{2}{*}{ MC1 } & SGF & 0.8892 & 0.8711 & $\underline{0.9531}$ & $\sim$ & 77.96 & \multirow{2}{*}{7.36} \\
\hline & SIF & NA & NA & NA & $\sim$ & 86.7 & \\
\hline \multirow{2}{*}{ MC2 } & SGF & 0.8961 & 0.7874 & $\underline{0.9777}$ & 0.3816 & 45.96 & \multirow{2}{*}{16.03} \\
\hline & SIF & NA & NA & NA & $\sim$ & 70.69 & \\
\hline \multirow{2}{*}{ MC3 } & SGF & 0.9244 & 0.8904 & $\underline{0.9474}$ & 0.2536 & 8.33 & \multirow{2}{*}{58.44} \\
\hline & SIF & NA & NA & NA & 0.2687 & 24.49 & \\
\hline \multirow{2}{*}{ MC4 } & SGF & 0.8851 & 0.8389 & $\underline{0.916}$ & 0.1585 & 4.69 & \multirow{2}{*}{77.25} \\
\hline & SIF & NA & NA & NA & 0.2392 & 17.35 & \\
\hline \multirow{2}{*}{ Delsym ${ }^{\circledR}$} & SGF & \multirow{2}{*}{${\underline{0.9962^{\mathrm{d}}}}$} & \multirow{2}{*}{0.8089} & \multirow{2}{*}{0.9318} & \multirow{2}{*}{0.7665} & 5.18 & \multirow[t]{2}{*}{$\sim$} \\
\hline & SIF & & & & & 16.09 & \\
\hline
\end{tabular}

a RE refers to the release efficiency calculated from areas under the curve by the trapezoidal method

${ }^{\mathrm{b}} f_{2}$ refers to the similarity factor

c NA means not applicable

d Calculated for the whole phase 
dosage form. Fig. 4 shows the rheological behavior of the alginate-based systems and revealed dependency of rheology on the shear rate where its values decreased upon the application of shear. The commercial product showed a similar rheological profile but the recorded viscosity values were higher than those of the tested alginate based systems. Similar rheological behavior was recorded for the alginate-based in situ gelling system (5). There was a slight increase in viscosity as a function of Eudragit S 100 concentration which may be due to increased amounts of dispersed particles.

Drug release pattern from different in situ gelling systems. - Release profiles of the drug from different in situ gelling systems are shown in Fig. 5. Calculated release efficiency values are presented in Table II.

The drug release profile from the $2 \%(\mathrm{~m} / \mathrm{V})$ alginate dispersion (A2) showed slow release in the gastric medium with release efficiency of $33.1 \%$. However, this formulation liberated the rest of the labeled drug at the early stage after shifting to $\mathrm{pH} 6.8$ (Fig. 5). This indicates that alginate can control drug release in the stomach only but liberates the entire amount very shortly after gastric emptying. This is further emphasized by calculating the release efficiency (RE) for drug release in the simulated intestinal fluid (SIF), which was increased to $83.1 \%$ (Table II). Failure of the pure alginate-based in situ gelling system to control the release of the drug after gastric emptying reflects the possibility of dose dumping. This highlights the need for improvement of such systems.

Accordingly, this study investigated the effect of using methacrylic resins to sustain drug release even after the rupturing of the gel matrix in the intestinal transit. This effect is attributed to the fact that cross-linked copolymers of methacrylic acid are ion exchangers and are able to bind drug cations (27).

Combination of Eudragit coated DM with the alginate based system resulted in a significant reduction in the drug release rate compared to the same alginate formulation containing the drug in the absence of Eudragit (A2). The release profiles indicated reduction in release efficiency after incorporation of the Eudragit coated drug in the alginate compared to that obtained from the pure drug loaded alginate. The extent of reduction in release efficiency increased with increasing the Eudragit concentration in the particles (Fig. 5). Reduction in drug release was evident both in acid and alkaline phases. Release of the drug from these in situ gel forming systems was characterized by an initial phase of retarded release that became higher on shifting to the intestinal phase. This biphasic pattern of release is a characteristic feature of matrix diffusion kinetics (28). The release efficiency for M1 was $25.56 \%$ in the gastric phase, which increased to reach $71.12 \%$ after $4 \mathrm{~h}$ in the intestinal phase expressing dose dumping. In contrast, M4 showed a release efficiency value of $1.07 \%$ in the gastric phase and $8.48 \%$ in the intestinal one, indicating a superior retarding effect for the release of the drug as Eudragit $S$ 100 concentration was increased (Table II). This significant decrease in the rate and extent of drug release with increasing Eudragit S 100 concentration can be attributed to the proposed hydrogen bonding that occurred between the $\mathrm{NH}^{+}$of the tertiary amine group of $\mathrm{DM}$ and the $\mathrm{C}=\mathrm{O}$ of the carboxylic moiety of Eudragit $\mathrm{S} 100$. An additional factor that plays a significant role in the drug release retardation is the gel barrier formed from the gelation of alginate. This latter effect of the alginate barrier can be observed in the slower release of the drug from the co-evaporates after being dispersed in the alginate system, M1, M2, M3 and M4, when compared to drug release from the co-evaporates alone in the absence of alginate MC1, MC2, MC3 and MC4 (Fig. 6). The percentage of drug 


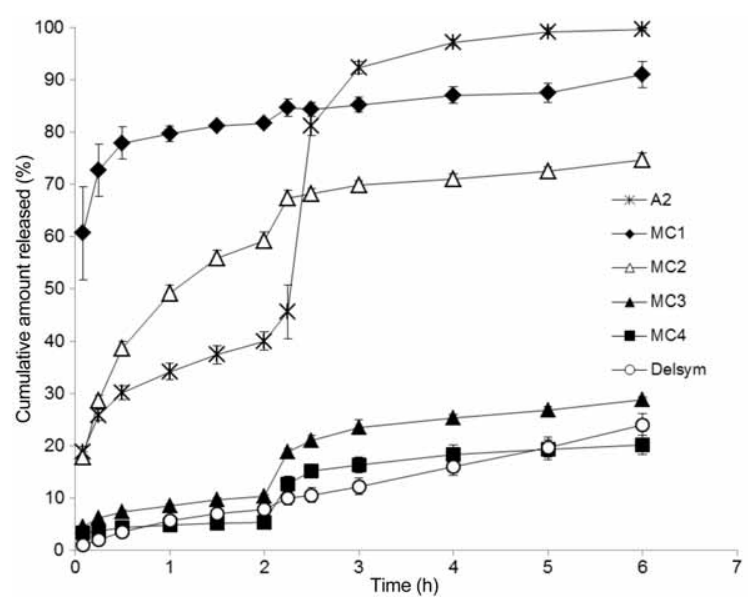

Fig. 6. In vitro release profiles of dextromethorphan hydrobromide from A2 (*), M1 (»), M2 ( $\square)$, M3 $(\boldsymbol{\Delta})$, M4 ( $\mathbf{\square})$ \& Delsym ${ }^{\circledR}$ suspension $(\bigcirc)$ plotted as cumulative \% released against time. Release was into simulated gastric fluid, $\mathrm{pH} 1.2$, for a period of $2 \mathrm{~h}$ and subsequently into simulated intestinal fluid, $\mathrm{pH} 6.8$, for a period of $4 \mathrm{~h}$. Each value is the mean $\pm \mathrm{SE}$ of three determinations.

release from all particles was significantly higher $(p<0.05)$ than that recorded after incorporation of the corresponding particles in the alginate vehicle. However, it should be noted that a particulate formulation like MC4 can provide a release profile similar to the commercial product Delsym ${ }^{\circledR}$, as discussed below.

Kinetic modeling of the release data. - AsAsSA As the release pattern is a biphasic one, each phase was studied separately to determine its kinetics. As shown in Table II, the correlation coefficient $\left(R^{2}\right)$ for the zero-order model ranges from 0.8754 to 0.9594 and from 0.785 to 0.982 for the gastric and intestinal phases, respectively, whereas that for the Higuchi model ranges from 0.916 to 0.9991 and from 0.7998 to 0.9934 for the gastric and intestinal phases, respectively, suggesting the Higuchi model (29) for drug release from dosage forms.

Drug release was further analyzed by the Korsmeyer-Peppas model to define the mechanism of drug release; $n$ values obtained were within the range of $0.15-0.50$, which are beyond the limits of the Krosmeyer model so-called power law.

Drug release kinetics of these formulations corresponds best to Higuchi's model and drug release mechanism as per $n$ value of Krosmeyer-Peppas (power law) cannot be predicted clearly, since it appears to be a complex mechanism of swelling, diffusion and erosion.

On the other hand, the commercial product $\left(\right.$ Delsym $\left.^{\circledR}\right)$ showed zero order release kinetics, as indicated by the recorded $R^{2}$. However, its $n$ value $(0.7665)$ indicates that the drug release from this ion exchange resin follows anomalous (non-Fickian) diffusion.

Similarity test. - As stated by FDA guidelines, two dissolution profiles are considered similar when the $f_{2}$ value is 50 by applying the $f_{2}$ similarity test as stated by FDA guidelines. The similarity factor test can be conducted using the following equation. 


$$
f_{2}=50 \times \log \left\{\frac{1}{\sqrt{\left[1+\left(\frac{1}{n}\right) \sum_{t=1}^{n}\left(R_{\mathrm{t}}-T_{\mathrm{t}}\right)^{2}\right]}} \times 100\right\}
$$

Where $n$ is the number of time points, $R_{\mathrm{t}}$ is the mean percent drug released of the reference formulation at time $t$ and $T_{\mathrm{t}}$ is the mean percent drug released of the test formulation at the same time.

The $f_{2}$ values are illustrated in Table II. The data indicate that formulations M2, M3, M4, MC3 and MC4 have dissolution profiles similar to the commercial product. These results confirm the suitability of the developed system to control drug release both under the gastric and intestinal conditions with no possibility of dose dumping.

\section{CONCLUSIONS}

Use of the alginate based in situ gelling formulation as a liquid oral controlled release system can lead to dose dumping after gastric emptying. Combination of the enteric coating technique with the in situ gelling system is a promising strategy to develop a liquid oral sustained release system with close control of the release rate both in the stomach and under intestinal conditions.

Acknowledgements. - The authors thank King Abdulaziz City for Science and Technology, Riyadh, Saudi Arabia, for funding the work with an acknowledgement to Kayyali chair of pharmaceutical industry for hosting the work.

\section{REFERENCES}

1. G. M. Jantzen and J. R. Robinson, Sustained and Controlled Release Drug Delivery Systems, Marcel Dekker, New York, 1996, pp. 575-610.

2. S. Miyazaki, H. Aoyama, N. Kawasaki, W. Kubo and D. Attwood, In situ-gelling gellan formulations as vehicles for oral drug delivery, J. Control. Release 60 (1999) 287-295; DOI: 10.1016/ S0168-3659(99)00084-X.

3. S. Miyazaki, W. Kubo and D. Attwood, Oral sustained delivery of theophylline using in situ gelation of sodium alginate, J. Control. Release 67 (2000) 275-280; DOI: 10.1016/s0168-3659(00) 00214-5.

4. S. Miyazaki, N. Kawasaki, W. Kubo, K. Endo and D. Attwood, Comparison of in situ gelling formulations for the oral delivery of cimetidine, Int. J. Pharm. 220 (2001) 161-168; DOI: 10.1016/ S0378-5173(01)00669-X.

5. W. Kubo, S. Miyazaki and D. Attwood, Oral sustained delivery of paracetamol from in situ-gelling gellan and sodium alginate formulations, Int. J. Pharm. 258 (2003) 55-64; DOI: 10.1016/ S0378-5173(03)00163-7.

6. D. Schmaljohann, Thermo- and pH-responsive polymers in drug delivery, Adv. Drug Deliv. Rev. 58 (2006) 1655-1670; DOI: 10.1016/j.addr.2006.09.020. 
7. T. Coviello, P. Matricardi, C. Marianecci and F. Alhaique, Polysaccharide hydrogels for modified release formulations, J. Control. Release 119 (2007) 5-24; DOI: S0168-3659(07)00039-9 [pii] 10.1016/j.jconrel.2007.01.004.

8. G. Rohith, B. K. Sridhar and A. Srinatha, Floating drug delivery of a locally acting H2-antagonist: an approach using an in situ gelling liquid formulation, Acta Pharm. 59 (2009) 345-354; DOI: 10.2478/v10007-009-0021-z.

9. L. Rodriguez, O. Caputo, M. Cini, C. Cavallari and R. Grecchi, In vitro release of theophylline from directly-compressed matrices containing methacrylic acid copolymers and/or dicalcium phosphate dehydrate, Farmaco 48 (1993) 1597-1604.

10. L. S. da Fonseca, R. P. Silveira, A. M. Deboni, E. V. Benvenutti, T. M. Costa, S. S. Guterres and A. R. Pohlmann, Nanocapsule@xerogel microparticles containing sodium diclofenac: a new strategy to control the release of drugs, Int. J. Pharm. 358 (2008) 292-295; DOI: S0378-5173(08)00118-X [pii] 10.1016/j.ijpharm.2008.02.005.

11. E. F. Reynolds James, Dextromethorphan Hydrobromide, Royal Pharmaceutical Society, London, 1996, p. 1066.

12. J. L. Bem and R. Peck, Dextromethorphan. An overview of safety issues, Drug Safety 7 (1992) 190-199.

13. E. S. Pender and B. R. Parks, Toxicity with dextromethorphan-containing preparations: a literature review and report of two additional cases, Pediatr. Emerg. Care 7 (1991) 163-165.

14. G. M. El Maghraby, Synergistic enhancement of itraconazole dissolution by ternary system formation with pluronic F68 and hydroxypropylmethylcellulose, Sci. Pharm. 77 (2009) 401-417; DOI: 10.3797/scipharm.0901-08.

15. F. K. Alanazi, M. El-Badry and I. A. Alsarra, Spray-dried HPMC microparticles of indomethacin impact of drug-polymer ratio and viscosity of the polymeric solution on dissolution, Saudi Pharm. J. 14 (2006) 100-107.

16. G. M. El Maghraby, E. M. Elzayat and F. K. Alanazi, Development of modified in situ gelling oral liquid sustained release formulation of dextromethorphan, Drug Dev. Ind. Pharm. (2011); DOI: 10.3109/03639045.2011.634811.

17. P. S. Rajinikanth and B. Mishra, Floating in situ gelling system of acetohydroxamic acid for clearance of H. pylori, Drug Dev. Ind. Pharm. 34 (2008) 577-587; DOI: 10.1080/03639040701831819

18. K. A. Khan, The concept of dissolution efficiency, J. Pharm. Pharmacol. 27 (1975) 48-49.

19. M. R. Louhaichi, S. Jebali, M. H. Loueslati, N. Adhoum and L. Monser, Simultaneous determination of pseudoephedrine, pheniramine, guaifenesin, pyrilamine, chlorpheniramine and dextromethorphan in cough and cold medicines by high performance liquid chromatography, Talanta 78 (2009) 991-997; DOI: 10.1016/j.talanta.2009.01.019.

20. F. Alanazi, H. Li, D. S. Halpern, S. Oie and D. R. Lu, Synthesis, preformulation and liposomal formulation of cholesteryl carborane esters with various fatty chains, Int. J. Pharm. 255 (2003) 189-197; DOI: S0378517303000887.

21. M. Malladi, R. Jukanti, R. Nair, S. Wagh, H. S. Padakanti and A. Mateti, Design and evaluation of taste masked dextromethorphan hydrobromide oral disintegrating tablets, Acta Pharm. 60 (2010) 267-280; DOI: 10.2478/v10007-010-0025-8.

22. R. P. Raffin, D. Jornada, S. Haas, A. R. Pohlmann and S. S. Guterres, "Pantoprazole sodium loaded controlled release microparticles," in 15th International Symposium on Microencapsulation, Parma, ed. (Italy, 2005), pp. 7-8.

23. K. H. Kim, M. J. Frank and N. L. Henderson, Application of differential scanning calorimetry to the study of solid drug dispersions, J. Pharm. Sci. 74 (1985) 283-289.

24. R. J. Fessenden and J. S. Fessenden, Fessenden and Fessenden Organic Chemistry, Brooks/Cole publishing company, Pacific Grove, 1990, pp. 323-390. 
25. Technical Information on EUDRAGIT L 100 and EUDRAGIT S 100, http:/ /eudragit.evonik.com/ product/eudragit/Documents/evonik-specification-eudragit-l-100-and-s-100.pdf; access date January 10, 2011.

26. M. R. Jenquin and J. W. McGinity, Characterization of acrylic resin matrix films and mechanisms of drug-polymer interaction, Int. J. Pharm. 101 (1994) 23-24.

27. K. O. R. Lehman, Chemistry and Application Properties of Polymethacrylate Coating Systems, Informa Healthcare USA, Inc., New York, 1990, pp. 101-174.

28. B. J. Lee, J. H. Cui, T. W. Kim, M. Y. Heo and C. K. Kim, Biphasic release characteristics of dual drug-loaded alginate beads, Arch. Pharm. Res. 21 (1998) 645-650.

29. T. Higuchi, Rate of release of medicaments from ointment bases containing drugs in suspension, J. Pharm. Sci. 50 (1961) 874-875.

30. G. M. Jantzen and J. R. Robinson, Sustained and Controlled Release Drug Delivery Systems, Marcel Dekker, New York, 1996, pp. 575-610.

31. S. Miyazaki, H. Aoyama, N. Kawasaki, W. Kubo and D. Attwood, In situ-gelling gellan formulations as vehicles for oral drug delivery, J. Control. Release 60 (1999) 287-295; DOI: 10.1016/ S0168-3659(99)00084-X.

32. S. Miyazaki, W. Kubo and D. Attwood, Oral sustained delivery of theophylline using in situ gelation of sodium alginate, J. Control Release 67 (2000) 275-280; DOI: 10.1016/s0168-3659(00)00214-5.

33. S. Miyazaki, N. Kawasaki, W. Kubo, K. Endo and D. Attwood, Comparison of in situ gelling formulations for the oral delivery of cimetidine, Int. J. Pharm. 220 (2001) 161-168; DOI: 10.1016/ S0378-5173(01)00669-X.

34. W. Kubo, S. Miyazaki and D. Attwood, Oral sustained delivery of paracetamol from in situ-gelling gellan and sodium alginate formulations, Int. J. Pharm. 258 (2003) 55-64; DOI: 10.1016/ S0378-5173(03)00163-7.

35. D. Schmaljohann, Thermo- and pH-responsive polymers in drug delivery, Adv. Drug Deliv. Rev. 58 (2006) 1655-1670; DOI: 10.1016/j.addr.2006.09.020.

36. T. Coviello, P. Matricardi, C. Marianecci and F. Alhaique, Polysaccharide hydrogels for modified release formulations, J. Control. Release 119 (2007) 5-24; DOI: S0168-3659(07)00039-9 [pii] 10.1016/j.jconrel.2007.01.004.

37. G. Rohith, B. K. Sridhar and A. Srinatha, Floating drug delivery of a locally acting H2-antagonist: an approach using an in situ gelling liquid formulation, Acta Pharm. 59 (2009) 345-354; DOI: $10.2478 / v 10007-009-0021-z$.

38. L. Rodriguez, O. Caputo, M. Cini, C. Cavallari and R. Grecchi, In vitro release of theophylline from directly-compressed matrices containing methacrylic acid copolymers and/or dicalcium phosphate dehydrate, Farmaco 48 (1993) 1597-1604.

39. L. S. da Fonseca, R. P. Silveira, A. M. Deboni, E. V. Benvenutti, T. M. Costa, S. S. Guterres and A. R. Pohlmann, Nanocapsule@xerogel microparticles containing sodium diclofenac: a new strategy to control the release of drugs, Int. J. Pharm. 358 (2008) 292-295; DOI: S0378-5173(08) 00118-X [pii] 10.1016/j.ijpharm.2008.02.005.

40. E. F. Reynolds James, Dextromethorphan Hydrobromide, Royal Pharmaceutical Society, London, 1996, p. 1066.

41. J. L. Bem and R. Peck, Dextromethorphan. An overview of safety issues, Drug Safety 7 (1992) 190-199.

42. E. S. Pender and B. R. Parks, Toxicity with dextromethorphan-containing preparations: a literature review and report of two additional cases, Pediatr. Emerg. Care 7 (1991) 163-165.

43. G. M. El Maghraby, Synergistic enhancement of itraconazole dissolution by ternary system formation with pluronic F68 and hydroxypropylmethylcellulose, Sci. Pharm. 77 (2009) 401-417; DOI: 10.3797/scipharm.0901-08. 
44. F. K. Alanazi, M. El-Badry and I. A. Alsarra, Spray-dried HPMC microparticles of indomethacin impact of drug-polymer ratio and viscosity of the Polymeric solution on dissolution, Saudi Pharm. J. 14 (2006) 100-107.

45. G. M. El Maghraby, E. M. Elzayat and F. K. Alanazi, Development of modified in situ gelling oral liquid sustained release formulation of dextromethorphan, Drug Dev. Ind. Pharm. (2011); DOI: 10.3109/03639045.2011.634811.

46. P. S. Rajinikanth and B. Mishra, Floating in situ gelling system of acetohydroxamic acid for clearance of H. pylori, Drug Dev. Ind. Pharm. 34 (2008) 577-587; DOI: 10.1080/03639040701831819.

47. K. A. Khan, The concept of dissolution efficiency, J. Pharm. Pharmacol. 27 (1975) 48-49.

48. M. R. Louhaichi, S. Jebali, M. H. Loueslati, N. Adhoum and L. Monser, Simultaneous determination of pseudoephedrine, pheniramine, guaifenesin, pyrilamine, chlorpheniramine and dextromethorphan in cough and cold medicines by high performance liquid chromatography, Talanta 78 (2009) 991-997; DOI: 10.1016/j.talanta.2009.01.019.

49. F. Alanazi, H. Li, D. S. Halpern, S. Oie and D. R. Lu, Synthesis, preformulation and liposomal formulation of cholesteryl carborane esters with various fatty chains, Int. J. Pharm. 255 (2003) 189-197; DOI: S0378517303000887.

50. M. Malladi, R. Jukanti, R. Nair, S. Wagh, H. S. Padakanti and A. Mateti, Design and evaluation of taste masked dextromethorphan hydrobromide oral disintegrating tablets, Acta Pharm. 60 (2010) 267-280; DOI: 10.2478/v10007-010-0025-8.

51. R. P. Raffin, D. Jornada, S. Haas, A. R. Pohlmann and S. S. Guterres, Pantoprazole sodium loaded controlled release microparticles, in 15th International Symposium on Microencapsulation, Parma, ed. (Italy, 2005), pp. 7-8.

52. K. H. Kim, M. J. Frank and N. L. Henderson, Application of differential scanning calorimetry to the study of solid drug dispersions, J. Pharm. Sci. 74 (1985) 283-289.

53. R. J. Fessenden and J. S. Fessenden, Fessenden and Fessenden Organic Chemistry, Brooks/Cole publishing company, Pacific Grove, 1990, pp. 323-390.

54. Technical Information on EUDRAGIT L 100 and EUDRAGIT S 100, http:/ /eudragit.evonik.com/ product/eudragit/Documents/evonik-specification-eudragit-1-100-and-s-100.pdf; access date January 10, 2011.

55. M. R. Jenquin and J. W. McGinity, Characterization of acrylic resin matrix films and mechanisms of drug-polymer interaction, Int. J. Pharm. 101 (1994) 23-24.

56. K. O. R. Lehman, Chemistry and application properties of polymethacrylate coating systems, Informa Healthcare USA, Inc., New York, 1990, pp. 101-174.

57. B. J. Lee, J. H. Cui, T. W. Kim, M. Y. Heo and C. K. Kim, Biphasic release characteristics of dual drug-loaded alginate beads, Arch Pharm. Res. 21 (1998) 645-650.

58. T. Higuchi, Rate of release of medicaments from ointment bases containing drugs in suspension, J. Pharm. Sci. 50 (1961) 874-875. 